\title{
Proposta alternativa para a adequação dos pequenos municípios à PNRS, baseada em sistema de produto-serviço
}

Jairo Pablo Alves de Carvalho americavend@gmail.com

Universidade Tecnológica Federa do Paraná - UTFPR, Curitiba, Paraná, Brasil

\section{Milton Borsato}

borsato@utfpr.edu.br

Universidade Tecnológica Federa do Paraná - UTFPR, Curitiba, Paraná, Brasil

\begin{abstract}
RESUMO
O Governo brasileiro lançou, em 2010, a Política Nacional de Resíduos Sólidos (PNRS), com o objetivo de estabelecer diretrizes e normatizar boas práticas de gerenciamento dos resíduos sólidos urbanos. Dentre as metas traçadas, estava a de acabar com os depósitos a céu aberto (lixões) em todos os municípios brasileiros. Contudo mais de um terço ainda não se adequou, mormente por não ter orçamento para custear as soluções tecnológicas disponíveis. Algumas administrações têm optado pelo consórcio intermunicipal para ganharem massa crítica e poderem utilizar as tecnologias existentes. Mas esta estratégia implica na transferência de passivo ambiental e social para o município sede do empreendimento, cujo território receptará o lixo dos demais, levando a desvalorização imobiliária na região. Uma solução utilizada com relativo sucesso em países asiáticos tem sido o uso de biodigestores de pequeno porte. Mas a solução apresenta alta taxa de abandono, devido a carência de serviços de suporte como treinamento de instaladores e usuários, atendimento ao consumidor, suporte técnico e manutenção. Este artigo apresenta a proposta dum sistema produto-serviço (PSS), projetado para mitigar as debilidades do modelo asiático, a qual elimina a etapa de transporte do lixo, como alternativa para os pequenos municípios brasileiros adequarem-se à PNRS.
\end{abstract}

PALAVRAS-CHAVE: PNRS. lixo orgânico. resíduos sólidos. PSS. Biodigestor. Biogás. 


\section{INTRODUÇÃO}

Nos países em desenvolvimento, o lixo orgânico doméstico é comumente destinado a lixões (RAMOS et al., 2017) - locais nos quais os resíduos são depositados diretamente sobre o solo, a céu aberto, sem nenhuma forma de tratamento (MONTEIRO, 2001). Como consequência, há uma série de problemas socio-ambientais, como a contaminação do ar e do solo, e a proliferação de doenças (OLIVEIRA; OLIVEIRA, 2013; UNNIKRISHNAN; SINGH, 2010).

No Brasil, a situação não é diferente. 0 país possui quase 3 mil lixões, distribuídos em 1600 cidades, os quais afetam a saúde de 95 milhões de pessoas. E mais de 3 bilhões de reais são gastos, por ano, com o tratamento de pessoas que ficam doentes por consequência da contaminação da água, solo e ar (BLAST ELAINE, 2018).

Cerca de metade do lixo produzido no país é orgânico (MMA, 2017). Esse, além dos problemas supra-mencionados, contribui também para a proliferação de agentes propagadores de doenças, como ratos, mosquitos e outros.

O governo brasileiro aprovou a lei 12.305/2010, que instituiu a Política Nacional de Resíduos Sólidos (PNRS), e estipulou a meta de acabar com os lixões em 2014 (AMBIENTE, [s.d.]; FERREIRA, 2018). Porém, a meta não foi cumprida. Em seguida o Senado Federal aprovou o projeto de lei no 425 de 2014, prorrogando os prazos para até 2021(BRASIL, 2010).

Conforme a Constituição Federal, compete aos municípios o prover o serviço de limpeza urbana. Contudo, segundo a Confederação Nacional dos Municípios, não há caixa disponível (FEDERAL, [s.d.]). Cerca de $90 \%$ deles são de pequeno porte, com menos de 50.000 habitantes, e não tem capacidade de gerar recursos para resolver os problemas de resíduos sólidos nem para buscar financiamento em organizações de fomento (FERREIRA, 2018). Portanto, para que os municípios cumpram a meta, é necessário idealizar e implementar soluções inovadoras para a destinação e tratamento do lixo orgânico, as quais sejam economicamente viáveis.

Há soluções tecnológicas disponíveis no mercado para fazer frente ao problema. Contudo, elas estão além do alcance dos municípios, atuais responsáveis por contratá-las, devido ao seu elevado custo. Além disso, apresentam também outras fragilidades, como o não contemplar outros aspectos do desenvolvimento sustentável, como a transferência de passivo ambiental (teleacoplamento) e, em especial o social, que inclui a participação ativa dos cidadãos na conservação do ambiente e valorização do território.

O presente artigo discute as fragilidades das soluções tecnológicas disponíveis sob a ótica do desenvolvimento territorial sustentável, com ênfase nas questões econômica e do uso e apropriação do território (territorialidade), e apresenta uma proposta de solução que contempla as lacunas observadas. Primeiramente, na seção 2, apresenta o conceito de sustentabilidade associado ao território. Em seguida, na seção 3 discute as soluções tecnológicas disponíveis. Então, na seção 4 , apresenta a proposta e na 5 tece as considerações finais. 


\title{
2 SUSTENTABILIDADE NO TERRITÓRIO
}

O conceito de desenvolvimento sustentável surgiu nas décadas de 1970 e 1980, sendo consolidado pela Comissão Mundial sobre o Meio Ambiente e Desenvolvimento (CMAD), criada pela Organização das Nações Unidas (ONU), também conhecida como Comissão Brundtland. A CMAD o definiu como

\begin{abstract}
Um processo de transformação no qual a exploração dos recursos, a direção dos investimentos, a orientação do desenvolvimento tecnológico e a mudança institucional se harmonizam e reforça o potencial presente e futuro, a fim de atender às necessidades e aspirações futuras (...) é aquele que atende às necessidades do presente sem comprometer a possibilidade das gerações futuras atenderem as suas próprias necessidades (WCED, 1987, p. 46).
\end{abstract}

Mais tarde o mesmo foi aprimorado por Sachs (2008), o qual propôs oito dimensões do desenvolvimento sustentável: ecológica, ambiental, social, cultural, econômica, política nacional, política internacional e territorial. Esse conceito é bem mais amplo do que o afamado "tripé da sustentabilidade", o qual contempla apenas as dimensões econômica, ambiental e social.

As dimensões ecológica e ambiental consistem-se na preservação do capital natural, intensificando o uso potencial dos recursos com um mínimo de danos aos sistemas, não retirando mais que sua capacidade de regeneração nem lançando mais que sua capacidade de absorção. A dimensão social enfoca o alcance de um patamar razoável de igualdade social, a distribuição de renda, emprego e acesso a serviços básicos e liberdade de expressão. A cultural relaciona-se com a preservação dos valores, permitindo aos atores locais protagonizar seu desenvolvimento, em oposição a uma mimetização dos países desenvolvidos. A econômica enfoca a alocação e gerenciamento mais eficiente dos recursos e fluxo constante de investimentos públicos e privados. A dimensão política nacional inclui a democracia e o desenvolvimento do Estado para implementar as políticas nacionais em parceria com os empreendedores com um nível razoável de coesão social. A dimensão política internacional inclui temas como o compartilhamento de responsabilidades, a cooperação econômica científica e tecnológica entre as nações, e o favorecimento dos parceiros mais fracos nas relações bi ou multilaterais. Finalmente, a dimensão territorial faz referência às configurações urbanas e rurais balanceadas, estratégias seguras de desenvolvimento para áreas ecologicamente frágeis e a superação das disparidades inter-regionais, entre outros (LAMIM-GUEDES, 2012; PUBLICADIREITO, [s. d.]).

Sachs (2008) trata também do conceito de territorialidade, considerando o aspecto relacional da dimensão territorial, associado à re-significação do espaço que as pessoas ocupam e sua nova identidade com ele. A territorialidade é o capital social construído no território. Segundo Fukuyama (1995), o capital social é "a capacidade das pessoas para trabalhar em conjunto para fins comuns em grupos e organizações". Assim pode-se dizer que a territorialidade está relacionada com a capacidade das pessoas para trabalhar em conjunto dentro dum território. Ou seja, de cooperarem em prol do bem comum numa localidade - por exemplo, o bairro ou cidade onde residem. E se esta ação comum aumenta o valor simbólico atribuído àquela localidade, o valor do território para os habitantes, e consequentemente sua disposição de cuidá-lo e mantê-lo, será maior. Daí observase um benefício social fruto da ação cooperativa. E conclui-se que soluções que 
contemplem o engajamento associativo dos cidadãos tendem à construção do capital social e valorização simbólica do território, aspectos importantes dentro das dimensões territorial e social da sustentabilidade.

Por outro lado, e agora voltando ao ponto focal do problema abordado neste artigo, o engajamento pode conduzir a um resultado oposto, principalmente em se tratando de soluções para a solução do problema de destinação do lixo orgânico. A escolha duma cidade como destino do lixo de outras pode conduzir a uma insatisfação popular e a redução do valor simbólico atribuído ao território, com impactos em outras dimensões como a econômica, com consequente desvalorização imobiliária na região. Cita-se, como exemplo, o caso do município de Mandirituba, o qual foi escolhido para receber o lixo de 17 cidades da região metropolitana de Curitiba em 2009, mesmo sob aberta manifestação de insatisfação da população (KUMEGAWA, 2018).

O caso acima nos traz algumas perguntas para consideração: Quais iniciativas podem ser enquadradas no conceito "desenvolvimento territorial sustentável", considerando também a dimensão territorial? Pode considerar-se uma iniciativa de desenvolvimento territorial como "sustentável" quando o resultado de sua aplicação é, no todo ou em parte, a transferência do impacto ambiental, econômico ou social para outro território?

O mundo de hoje é interconectado, ou "tele-acoplado". Desconsiderar o efeito do tele-acoplamento conduz a trabalhar-se em soluções de redução de degradação ambiental numa dada localidade, as quais resultarão em aumento da mesma em outras regiões. Em virtude das consequências do tele-acoplamento, a sustentabilidade ambiental urbana foi inclusa como um pilar na agenda urbana dos membros das Nações Unidas. Entende-se que a governança urbana deverá considerar o tele-acoplamento e incluir a aculturação ambiental e o direcionamento de incentivos aos cidadãos com foco na preservação planetária, e não somente localizada (IBPES, 2018). Daí depreende-se que o desenvolvimento de quaisquer tecnologias para a solução do problema de processamento dos resíduos sólidos orgânicos num dado território deveria levar em consideração possíveis efeitos de tele-acoplamento e evitá-los, se possível. Infelizmente, isso ainda não acontece, como veremos na próxima seção.

\section{SOLUÇÕES TECNOLÓGICAS DISPONÍVEIS}

Para fazer frente ao problema da destinação dos resíduos sólidos, o governo brasileiro atuou na dimensão política aprovando a lei 12.305/2010, que instituiu a Política Nacional de Resíduos Sólidos (PNRS), e traçou a meta de acabar com os lixões em 2014 (FERREIRA, 2018). Porém, a meta não foi cumprida. Em 2015, 41,3\% do lixo foi descartado de maneira inapropriada, em lixões ou aterros sem qualquer espécie de tratamento (GREICE et al., 2017). Como medida paliativa, o Senado Federal aprovou o projeto de lei no 425 de 2014, prorrogando os prazos para até 2021 (BRASIL, 2010).

De acordo com a Constituição Federal, o serviço de limpeza urbana deve ser provido pelos municípios. Contudo, segundo a Confederação Nacional dos Municípios, não há caixa disponível. Seriam necessários $\mathrm{R} \$ 70$ bilhões para que todos os municípios cumprissem a lei (FEDERAL, [s.d.]). 
As tecnologias oferecidas pela iniciativa privada estão além do orçamento dos municípios. Segundo ZURBRUUG (2003), nos países em desenvolvimento as cidades não dispõem de recursos suficientes para atender a demanda de coleta de lixo. Isso porque elas estão em constante crescimento devido ao aumento da população, e as taxas cobradas não geram caixa suficiente para prover um nível adequado do serviço. Cita-se, como exemplo, a cidade de Curitiba, considerada vanguardista em iniciativas ecológicas. Em 2017, o custo do serviço de gerenciamento de lixos sólidos urbanos, para o município, foi de R\$238 milhões, contra R\$118 milhões de arrecadação (GAZETA DO POVO, 2018).

ZURBRUUG (2003) também aclara que, usualmente, as soluções propostas são aquelas aplicadas nos países ricos. Contudo, essas são baseadas em padrões tecnológicos e práticas adequadas às condições e ambiente regulatório daqueles países, e não levam em consideração as diferenças técnicas, econômicas, sociais e institucionais dos países em desenvolvimento.

Atualmente, os métodos de tratamento considerados como alternativas viáveis no cenário nacional são a compostagem, a recuperação energética, a reciclagem e a disposição em aterros sanitários (ABRELPE, 2015). Apresentamos, a seguir, quadros quantitativos de custos de cada solução, para análise, com dados levantados pela Associação Brasileira de Empresas de Limpeza Pública e Resíduos Especiais (ABRELPE), para cada um dos sistemas, e uma breve análise de sua viabilidade. As avaliações consideram a geração de 1,04kg de lixo por habitante / dia, (ABRELPE, 2016), e um mês de 30 dias. A Tabela 1 traz os custos da tecnologia de compostagem.

Tabela 1 - Custos de instalação e operação para unidades de compostagem

\begin{tabular}{|c|c|c|}
\hline \multicolumn{3}{|c|}{ Compostagem } \\
\hline Faixa de população & Custos de instalação & Custos operação \\
\hline 30 a 250 mil & $\mathrm{R} \$ 3 /$ ton & $\mathrm{R} \$ 90 /$ ton \\
250 mil a 1 milhão & $\mathrm{R} \$ 5,5 /$ ton & $\mathrm{R} \$ 70 /$ ton \\
\hline mais de 1 milhão & $\mathrm{R} \$ 3,075 /$ ton & $\mathrm{R} \$ 45 /$ ton \\
\hline
\end{tabular}

Fonte: BNDES, 2014 apud. ABRELPE, 2015

Pelos dados acima, teríamos uma despesa de operação de $R \$ 84.240$ mensais com compostagem, para um município de $\mathbf{3 0 . 0 0 0}$ habitantes, fora os custos com instalação. Para efeitos de comparação com a realidade dum município pequeno, levantou-se informações, numa entrevista presencial, com funcionários da secretaria da agricultura do município de Contenda - PR, sobre o orçamento atual para o gerenciamento de resíduos sólidos. O município possui população próxima de 20.000 habitantes. O orçamento do município para o tratamento do lixo é de $\mathrm{R} \$ 15.000,00$ mensais, o que equivaleria a $\mathrm{R} \$ 22.500,00$ para 30 mil habitantes. Este valor é cerca de um quarto do necessário para o gerenciamento utilizando-se a tecnologia. Os custos podem ser mitigados pela venda do fertilizante produzido pela compostagem. Contudo, é necessário existir demanda pelo produto no município, o que geralmente não ocorre quando as atividades industriais existentes em seu perímetro são predominantemente urbanas. Na Tabela 2, apresentam-se os custos para instalação e operação de aterros sanitários. 
Tabela 2 - Custos para implantação e operação de aterros sanitários

\begin{tabular}{|c|c|c|c|c|}
\hline Porte & $\begin{array}{c}\text { Toneladas } \\
\text { processadas / dia }\end{array}$ & Custos de instalação & $\begin{array}{c}\text { Custos } \\
\text { operação }\end{array}$ & Total \\
\hline Pequeno & 100 & 6.976 .285 & 45.468 .163 & 52,4 milhões \\
\hline Médio & 800 & 30.049 .713 & 206.485 .324 & 236,5 milhões \\
\hline Grande & 2000 & 64.300 .115 & 461.494 .052 & 525,8 milhões \\
\hline
\end{tabular}

Fonte: ABETRE \& FGV, 2009 apud. ABRELPE, 2015

Observa-se que o custo de implantação dos aterros sanitários é alto. Portanto, é necessário haver produção de lixo em grande escala para viabilizar sua operação. Considerando o volume médio per capita de lixo diário gerado, um aterro de pequeno porte, com capacidade de 100 toneladas / dia, atenderia uma cidade de 100 mil habitantes. Ocorre que aproximadamente $95 \%$ dos municípios brasileiros têm população abaixo de 100 mil habitantes (ABRELPE, 2016). Para estes, o uso da tecnologia de aterros somente se tornaria viável mediante a realização de consórcios com municípios próximos.

A situação altera-se para municípios maiores. O custo total por capacidade de processamento diário pula de $R \$ 0,52$ milhões / toneladas / dia para plantas de porte pequeno para $\mathrm{R} \$ 0,26$ milhões / toneladas / dia para aterros de grande porte. A Tabela 3 apresenta os custos para estações de tratamento térmico.

Quadro 3 - Custos para implantação e operação de unidades de tratamento térmico

\begin{tabular}{|c|c|c|}
\hline Capacidade máxima & Instalação & Operação \\
\hline 650 ton / dia & $\mathrm{R} \$ 280$ milhões & $\mathrm{R} \$ 23$ milhões \\
1300 ton / dia & $\mathrm{R} \$ 480$ milhões & $\mathrm{R} \$ 40,33$ milhões \\
\hline
\end{tabular}

Fonte: Adaptado de ABRELPE, 2015

Baseando-se nas mesmas premissas de geração de lixo per capita, a menor unidade de tratamento térmico apresentada no quadro atenderia um município de aproximados 1,3 milhões de habitantes. Esta tecnologia seria, portanto, uma alternativa a ser considerada apenas no caso das maiores cidades brasileiras. Para as demais, a alternativa é buscar o consórcio com outras de maior porte. O que já é realizado em algumas regiões, como a região metropolitana de Curitiba.

Contudo, o consorciamento entre os municípios para valer-se de uma das tecnologias mencionadas acarreta outro problema, que impacta na dimensão territorial da sustentabilidade. A infra-estrutura de processamento terá que ser instalada num território pertencente a um dos consorciados, o que significa que ele receberá o lixo dos demais municípios. E o território receptador sofrerá, por consequência, impactos negativos, tanto ambientais quanto sociais.

É o caso, por exemplo, do aterro do Caximba, situado em Curitiba, Paraná, o qual recebeu, durante 21 anos, além do lixo de Curitiba, o de outras 17 cidades da Região Metropolitana. Mesmo 6 anos após o seu fechamento, a região sofria com a desvalorização imobiliária e o mau odor dos gases provenientes do lixo (GLOBO, 2016). A diferença de densidade urbana entre a vizinhança imediata ao local do aterro e os arredores é visível ainda hoje (2019), como se observa pela Figura 1. 
Figura 1 - Imagem aérea do aterro do Caximba e regiões vizinhas

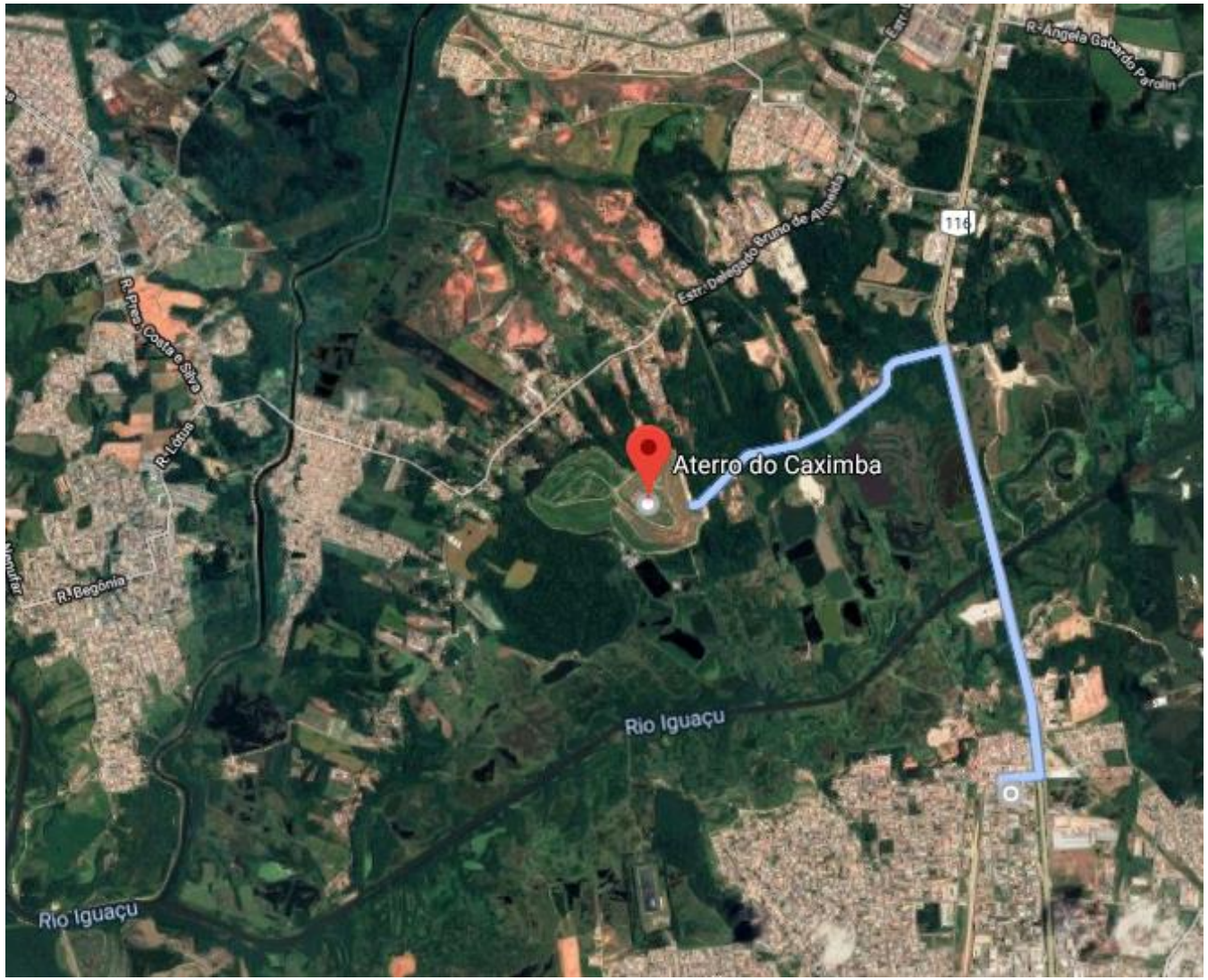

Fonte: Google (2019).

Neste caso, tem-se claramente caracterizada uma situação de teleacoplamento, onde a solução adotada por um território impacta negativamente noutro. E, quando considerada sob a ótica das dimensões da sustentabilidade segundo Sachs (2008), não se pode afirmar que a solução é sustentável.

Assim, considerando que as soluções tecnológicas disponíveis não oferecem a todos os municípios a possibilidade de promoverem o desenvolvimento territorial sustentável, remanesce o desafio de buscar-se soluções mais sustentáveis para a resolução do problema. Fruto da identificação desta lacuna, vislumbra-se a possibilidade duma solução a partir da adaptação de uma tecnologia já usada, com relativo sucesso, noutros países em desenvolvimento, a qual será apresentada na próxima seção.

\subsection{Sistema de produto serviço (PSS)}

O PSS surgiu como a maturação do entendimento de um conceito gradualmente introduzido primeiramente pelas empresas americanas. Na década de 90 , buscando recuperar sua performance e competitividade internacional, elas evoluíram do modelo tradicional: "fabricar e vender produtos", para "fabricar e financiar", "reparar e manter" e outras propostas de serviços associadas a produtos. Mais tarde, a United Nations Environmental Programme (UNEP) realizou um projeto de pesquisa analisando casos de aplicação do conceito em várias partes do mundo, e publicou um relatório intitulado: "Product-Service Systems and Sustainabillity" (KANDA; NAKAGAMI, 2006). 
PSS é um conceito que engloba a entrega de produtos e serviços para satisfazer as necessidades dos clientes (HAASE, 2017). Embora não haja uma definição consensual sobre o seu significado na comunidade científica, as duas proposições mais aceitas são:

i - "um sistema de produtos, serviços, redes de suporte e infra-estrutura projetada para ser: competitiva, satisfazer a necessidade dos consumidores e ter um menor impacto ambiental que os modelos de negócio tradicionais" (MONT, 2002);

ii - "um conjunto de produtos e serviços comercializável capaz de, conjuntamente, suprir a necessidade do usuário" (GOEDKOOP, M. J., VAN HALEN, C. J. G., TE RIELE, H. R. M., ROMMENS, 1999).

Tukker, (2004), dividiu as diferentes propostas de PSS existentes em 8 subdivisões, e as agrupou em 3 categorias de modelos de negócios - PSS orientada a produtos, PSS orientado ao uso e PSS orientado a resultados. No PSS orientado a produtos, o mesmo é vendido ao cliente, porém acompanhado de serviços como garantia e assistência técnica ao usuário. No PSS orientado a resultados o cliente paga, não pelo tempo, mas pelo resultado entregue através produto - exemplo: grama cortada. Neste caso, o tempo de disponibilidade da máquina de cortar grama é irrelevante para o cliente.

No PSS orientado ao uso o cliente paga pelo direito de uso ou disponibilidade do produto, por uma fração de tempo, rodagem ou outra unidade de medida. Mas propriedade do mesmo permanece sendo do fornecedor. A Figura 1 ilustra fluxo de produtos no modelo.

Figura 1 - Fluxo de produtos no modelo de negócio - PSS orientado ao uso

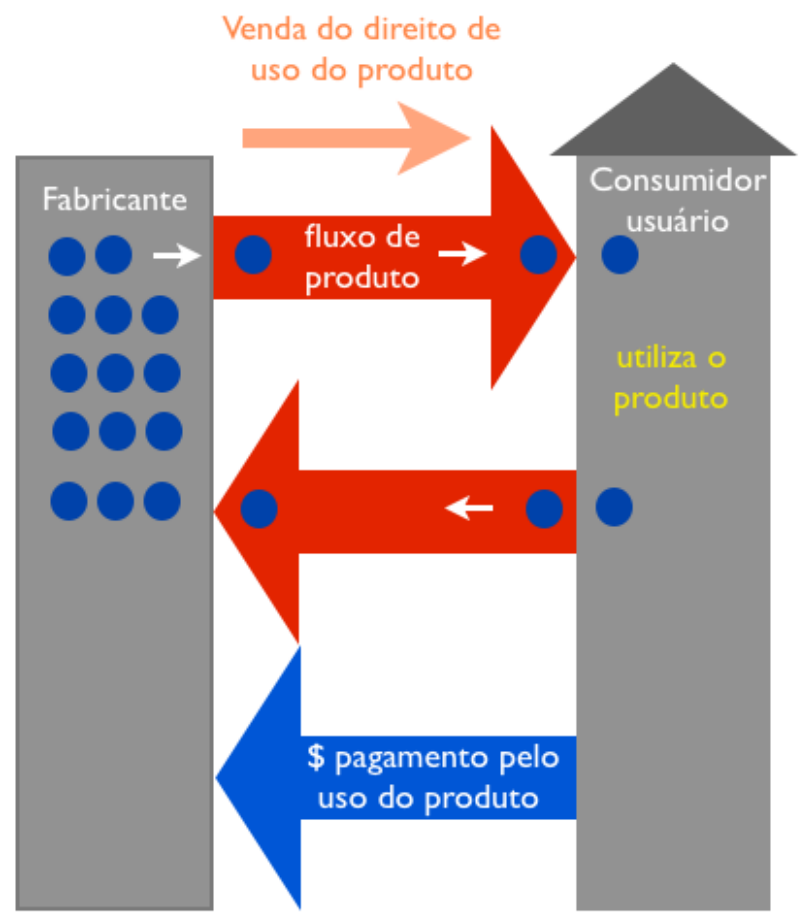

Fonte: os autores. 
Um exemplo deste modelo são os patinetes para locação por tempo determinado oferecidos por empresas privadas em várias grandes cidades do Brasil, das empresas GREEN e YELLOW. O PSS orientado ao uso permite que um usuário o qual não teria recursos para pagar pela aquisição do produto possa usufrui de sua funcionalidade. Devido a esta característica, afigura-se promissor para solucionar o problema atual dos municípios pequenos para atenderem as metas da PNRS, como se verá na seção 4, a seguir.

\section{PSS: UMA SOLUÇÃO POTENCIALMENTE VIÁVEL}

Segundo Carvalho, Bastchen e Borsato (2018), o estudo de soluções para a mitigação dos problemas relacionados com a destinação do lixo doméstico é uma tendência. Considerando o gerenciamento do lixo como modelo de negócio, verifica-se que as pesquisas atuais abordam questões relacionadas ao retorno sobre o investimento em tecnologias comerciais de transformação do lixo em combustível ou caracterização do mesmo para permitir melhor segregação e reaproveitamento de partes não combustíveis (ERIKSSON et al., 2014; HEISKANEN e JALAS, 2000; JAGTAP; RAHIMIFARD; KINGDOM, 2017; RAABE et al., 2017; SAHIMAA et al., 2015; SHAHBAZI et al., 2016; VAN EWIJK; STEGEMANN, 2016; YAZAN, 2016). Contudo, não abordam a problemática da eventual falta de capacidade dos municípios de custear o investimento inicial necessário para a implantação das tecnologias disponíveis.

A tecnologia mais adequada a ser utilizada depende da composição do lixo (BEYENE; WERKNEH; AMBAYE, 2018). Em países pobres e em desenvolvimento, a maior parcela do lixo é orgânica e biodegradável. No Brasil, o lixo orgânico monta $51,4 \%$ do total (GREICE et al., 2017). Neste caso, as soluções mais viáveis são aterros sanitários e a digestão anaeróbia, com o uso de biodigestores para a produção de biogás, devido ao impacto ambiental considerado baixo (BEYENE; WERKNEH; AMBAYE, 2018). A digestão anaeróbia para a produção de biogás é a tecnologia que apresenta maior viabilidade técnica e econômica de implementação (CHATTERJEE; MAZUMDER, 2016). Contudo, devido aos investimentos necessários a sua adoção, as soluções comerciais não estão acessíveis aos pequenos municípios brasileiros.

Neste contexto, enxerga-se uma luz no fim do túnel. Quando o Estado brasileiro instituiu a Política Nacional de Resíduos Sólidos, delineou, como um dos instrumentos para o seu cumprimento a parceria público privada, na seguinte diretiva:

Art. 8o São instrumentos da Política Nacional de Resíduos Sólidos, entre outros:

...VI - a cooperação técnica e financeira entre os setores público e privado para o desenvolvimento de pesquisas de novos produtos, métodos, processos e tecnologias de gestão, reciclagem, reutilização, tratamento de resíduos e disposição final ambientalmente adequada de rejeitos; (BRASIL, 2010).

A parceria público-privada pode ser eficaz para inovar, adaptando uma solução usada noutros países para a realidade dos municípios brasileiros. Biodigestores pequenos para tratamento do lixo orgânico são usados, com relativo sucesso, em países asiáticos. Principalmente em localidades afastadas do 
município, onde a economia de custos com coleta seria maior. Esses geram o biogás, que pode ser utilizado em fogões para cocção de alimentos ou geração de calor, e fertilizantes utilizáveis na agricultura. China e Índia, somente, somam mais de 30 milhões de sistemas instalados (ORTIZ; TERRAPON-PFA; DIENST, 2017). A tecnologia tem sido introduzida também em países africanos, com o apoio de organizações não governamentais (CLEMENS et al., 2018; LWIZA et al., 2017; ORSKOV et al., 2014; SMITH; SCHROENN; BLIGNAUT, 2014).

A tecnologia do biogás aplicada ao uso doméstico é a mais barata, e por isso mais utilizada em países pobres e em desenvolvimento em todo o mundo, objetivando o aproveitamento do lixo orgânico para a geração de energia e melhoramento das condições de vida das populações. No mundo todo são mais de 40 milhões de sistemas instalados (CHATTERJEE; MAZUMDER, 2016; ORTIZ; TERRAPON-PFA; DIENST, 2017).

É, portanto, potencialmente promissora para o cenário dos mais de $90 \%$ municípios brasileiros, os quais são pequenos e não dispõem de recursos suficientes para custear soluções para o gerenciamento adequado do lixo.

Outro aspecto que recomenda a tecnologia é o fato de ela eliminar a maior parcela do custo atual com gerenciamento de do lixo. Cerca de $70 \%$ do custo do gerenciamento de resíduos sólidos, é composto pelo transporte (GAZETA DO POVO, [s.d.]). Os biodigestores processam o lixo no local onde é gerado, eliminando a etapa do transporte.

Contudo, a solução também possui algumas deficiências. O custo inicial de aquisição ainda é relativamente alto para as famílias suportarem. E muitas, após a aquisição, deixam de utilizar a tecnologia devido à inabilidade de repará-los, a falta de acesso à compra de componentes roubados ou estragados. Outra causa de abandono do sistema é a compra da propriedade por pessoas que não conhecem nem acreditam na tecnologia, ou a saída, do domicílio, das pessoas que tinham treinamento para operar os biodigestores (CLEMENS et al., 2018; LWIZA et al., 2017; ORTIZ; TERRAPON-PFA; DIENST, 2017).

Lwiza et al. (2017), sugerem que as empresas e agências que promovam o biogás levantem previamente as necessidades e comportamento dos clientes dados quanto aos recursos necessários em termos de insumos para o biodigestor, mão de obra disponível para sua operação e disponibilidade de água, antes de implementar programas para a adoção da tecnologia. Acrescentam que as empresas de construção de sistemas de biogás devem fornecer informação, treinamento e serviço pós-venda para minimizar as ocorrências de malfuncionamento, e fazer a conexão entre as propriedades que adotarem o sistema e o mercado de acessórios de biogás.

Também identificaram que uma das causas do abandono da tecnologia pelos usuários é a não compatibilidade dos biodigestores com a necessidade dos usuários em termos de demanda por gás, recursos disponíveis no local para enchêlos e levá-los à condição operacional e disponibilidade de mão de obra. Outro fator é a exigência de se adquirir as tubulações e um fogão específico, ou adaptar o existente. Usando o biogás, os componentes se deterioram rapidamente, exigindo manutenção e troca. (ORTIZ; TERRAPON-PFA; DIENST, 2017).

Considerando as necessidades identificadas, vislumbra-se que uma solução potencial para as barreiras encontradas através de uma solução PSS orientada ao 
uso oferecida por empresas privadas para as autarquias públicas. No modelo proposto, o município pagaria pelo tempo de uso dos biodigestores. O fornecedor privado ofereceria treinamento aos potenciais usuários, serviços de instalação, transporte e manutenção dos equipamentos, conforme o esquema da Figura 2:

Figura 2 - PSS orientado ao uso proposto para municípios atenderem as metas da PNRS

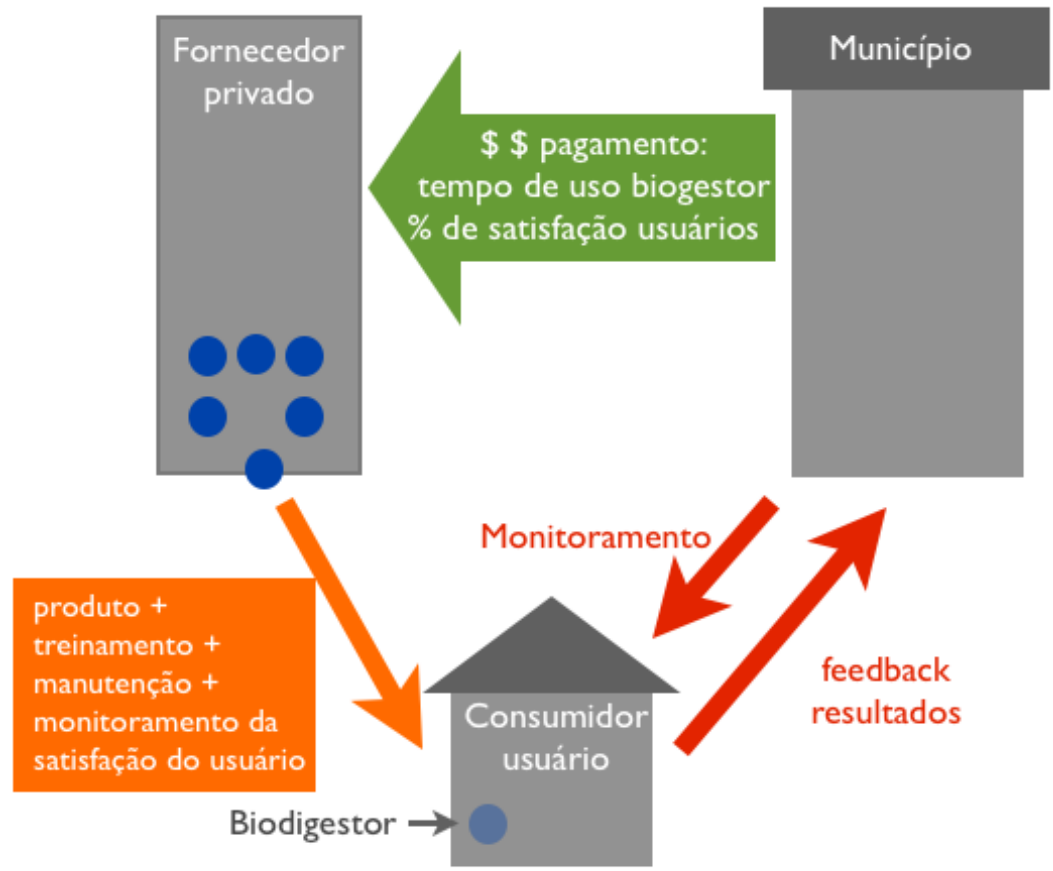

Fonte: Os autores.

O modelo proposto atende as demandas do usuário final identificadas, de treinamento de capacitação para uso dos biodigestores e suporte ao uso e manutenção ao longo do tempo. Também contempla a redução de custo de implantação, imposta pela escassez de verba municipal. O município não teria o custo de adquirir o equipamento. Apenas pagaria uma fração do mesmo, mensalmente, pelo uso. Para isso, pode alocar a verba atualmente investida no gerenciamento dos resíduos orgânicos, a fim de remunerar o fabricante. O modelo de negócio se viabiliza se mostrar-se rentável para o fornecedor privado, considerando seus custos de equipamento, pessoal e infra-estrutura de suporte para os consumidores finais.

O projeto pode ainda ser conjugado com outros, como o de horta nas casas, posto que um dos sub-produtos dos biodigestores é o fertilizante. A proposta aqui apresentada constitui-se numa alternativa de desenvolvimento efetivamente sustentável no tratamento de resíduos sólidos orgânicos. Isso porque, neste caso, o resíduo é tratado no mesmo local onde é produzido, e os subprodutos do processo são todos aproveitados no próprio local de tratamento, sem causar impactos negativos ao meio ambiente local nem reflexos noutros territórios. Esta situação está mais alinhada com a proposta de desenvolvimento ambiental sustentável, considerando-se as oito dimensões propostas por Sachs. 


\section{CONSIDERAÇÕES FINAIS}

O governo brasileiro aprovou a Política Nacional de Resíduos Sólidos em 2010, a qual inclui a meta de acabar com os lixões. Contudo, a responsabilidade do gerenciamento e custeio das soluções para o tratamento do lixo recai sobre os municípios. E esses se deparam com a falta de recursos para custear as soluções tecnológicas para o tratamento do lixo orgânico doméstico oficialmente reconhecidas como viáveis para o cumprimento da meta. Sobra, como alternativa, a consorciação, a qual, por sua vez, acarreta problemas de acúmulo de lixo de diversos municípios no território de um dos consorciados, conduzindo à depreciação do território receptador, bem como a problemas sociais.

Como uma alternativa potencial para a solução do problema foi sugerida a adoção da tecnologia de biodigestores de pequeno porte, uma solução já utilizada em muitos países da Ásia e África. Embora de baixo custo, mesmo esta solução apresenta algumas deficiências. Contudo, ao observar-se a natureza das mesmas, conclui-se que a proposta de realização dum programa de parceira público-privada para a contratação de PSS orientado ao uso de biodigestores de pequeno porte, a qual integre as etapas de treinamento, instalação, manutenção e suporte ao usuário mostra-se promissora para suprir as deficiências encontradas nos projetos de implantação realizados noutros países. Sendo este o caso, teria-se uma situação na qual o lixo doméstico é disposto e tratado no local de sua produção, e os subprodutos do processo também são aproveitados no mesmo lugar. Tal solução contribuiria efetivamente para o desenvolvimento sustentável, considerando-se a dimensão territorial da sustentabilidade.

A viabilidade da presente proposta, para os atores envolvidos, deve ser analisada considerando-se o caso de cada município. No presente, está avaliandose a mesma para um pequeno município do estado do Paraná. Sugere-se, para trabalhos futuros, a análise de viabilidade para implantação da mesma em municípios localizados noutras regiões do Brasil. 


\title{
Alternative proposal for the adaptation of small municipalities to the PNRS, based on a product-service system
}

\begin{abstract}
Brazilian government launched, on 2010, the National Solid Waste Politic (PNRS), with the objective of to establish guidelines and to normatize good practices of urban solid residues management. Among the goals traced, was that of to finish with the dumping grounds in all brazilian counties. However, more than one third of them didn't adequate themselves, mainly for lack of budget to pay for the technological solutions available. Some choosed to consortiate with others to gain critical mass and could use the existent technologies. But this strategy implicates in the trasnfer of the social and environmental passive to the city which receives the enterprise, and whose territory will receive the waste from others, bringing a real state devaluation in that region. One solution used with some success in asian countries has been the use of small biodigesters. Nevertheless it presents high abandonment rates, due to the lack of support services like training of users and installers, customer attendance, technical support and maintenance. This article presents a proposal of a product-service system (PSS), projected to mitigate the weaknesses of the asian model, which erase the waste transportation stage, as an alternative to the small brasilian cities adequate themselves to the PNRS.
\end{abstract}

KEYWORDS: PNRS. organic waste. solid residues. PSS. Biodigester. Biogas. 


\section{REFERÊNCIAS}

ABRELPE. Estimativas dos Custos para Viabilizar a Universalização da Destinação Adequada de Resíduos Sólidos no Brasil. Disponível em:

<http://abrelpe.org.br/arquivos/pub_estudofinal_2015.pdf>. Acesso em 21 set. 2018.

ABRELPE. Panorama Dos Resíduos Sólidos No Brasil 2016. Disponível em: <www.abrelpe.org.br/Panorama/panorama2016.pdf>. Acesso em: 21 set. 2018.

AMBIENTE, M. DO M. Política Nacional de Resíduos Sólidos. Disponível em: <http://www.mma.gov.br/política-de-resíduos-sólidos>. Acesso em: 23 set. 2018.

BEYENE, H. D.; WERKNEH, A. A.; AMBAYE, T. G. Current updates on waste to energy ( WtE ) technologies : a review. Reinforced Plastics, v. 24, n. March, p. 111, 2018.

BLAST ELAINE. Brasil tem quase 3 mil lixões em 1.600 cidades, diz relatório | Natureza | G1. Disponível em:

<https://g1.globo.com/natureza/noticia/2018/09/14/brasil-tem-quase-3-millixoes-em-1600-cidades-diz-relatorio.ghtml>. Acesso em: 13 mai. 2019.

BRASIL, L. N. . 12. 30. DE 2 DE AGOSTO DE 2010. Política Nacional de Resíduos Sólidos. Disponível em: <http://www.planalto.gov.br/ccivil_03/_ato20072010/2010/lei/l12305.htm>. Acesso em: 13 mai. 2019.

CARVALHO, J.; BASTCHEN, G.; BORSATO, M. METHODS FOR SUPPORTING THE PROSPECTION OF OPPORTUNITIES AND THE FEASIBILITY ANALYSIS OF THE REUSE OF WASTE - OPPORTUNITIES AND TRENDS A LITERATURE REVIEW. (X. Xu, M. I. Dessouky, R. Y. Zhong, Eds.)48th International Conference on Computers \& Industrial Engineering 2018 (CIE48). Anais...Auckland, New Zeland: Curran Associates, Inc. (2019), 2018

CHATTERJEE, B.; MAZUMDER, D. Anaerobic digestion for the stabilization of the organic fraction of municipal solid waste : A review. v. 459, n. October 2015, p. 426-459, 2016.

CLEMENS, H. et al. Energy for Sustainable Development Africa Biogas Partnership Program : A review of clean cooking implementation through market development in East Africa. Energy for Sustainable Development, v. 46, p. 23-31, 2018. 
FEDERAL, S. De onde tirar recursos para pagar a limpeza pública - Revista Em Discussão! Disponível em:

<https://www12.senado.leg.br/emdiscussao/edicoes/residuos-solidos/realidadebrasileira-na-pratica-a-historia-e-outra/de-onde-tirar-recursos-para-pagar-alimpeza-publica>. Acesso em: 13 mai. 2019.

FERREIRA, A. Management of municipal solid waste in municipalities of Paraná. Revista Capital Científico - Eletrônica, v. 16, n. 2, 2018.

FUKUYAMA, F. Trust: the social virtues and the creation of prosperity. Free Press Paperbacks, 1995.

GAZETA DO POVO. Capital ecológica? Curitiba coleta e transporta o seu lixo como nos anos 80. Disponível em:

$<$ https://www.gazetadopovo.com.br/politica/parana/capital-ecologica-curitibacoleta-e-transporta-seu-lixo-como-nos-anos-80-c8z10l8tjx3m7q00azuee2f91/>. Acesso em: 13 mai. 2019.

GAZETA DO POVO. Que saco... é pra pôr o lixo? Saiba o que muda com a nova coleta | Gazeta do Povo. Disponível em:

$<$ https://www.gazetadopovo.com.br/vida-e-cidadania/que-saco-e-pra-por-o-lixosaiba-o-que-muda-com-a-nova-coleta-0mxts7z9qq081e4tn2yg4mll8/>. Acesso em: 13 mai. 2019.

GLOBO. Moradores sentem reflexo do lixo, 6 anos após o fechamento do caximba. Disponível em:

<http://g1.globo.com/pr/parana/noticia/2016/08/moradores-sentem-reflexo-dolixo-6-anos-apos-fechamento-da-caximba.html>. Acesso em 23 ago. 2019.

GOEDKOOP, M. J., VAN HALEN, C. J. G., TE RIELE, H. R. M., ROMMENS, P. J. M. No Title. Product service systems, ecological and economic basics, 1999.

GOOGLE. Aterro do Caximba - Campo de Santana. Disponível em: < https://www.google.com.br/maps/place/Aterro+do+Caximba/@-25.6181451,49.3612441,6950m/data=!3m1!1e3!4m19!1 m13!4m12!1m6!1m2!1s0x94dcfe4f9 0dd2ab9:0x2a81f2dde68fc5a9!2sAterro+do+Caximbat+Campo+de+Santana,+Curitiba+-+PR!2m2!1d-49.3366458!2d25.6197477 ! $1 \mathrm{~m} 3$ ! $2 \mathrm{~m} 2$ ! $1 \mathrm{~d}-49.3150405$ !2d25.6352137!3e0!3m4!1s0x94dcfe4f90dd2ab9:0x2a81f2dde68fc5a9!8m2!3d25.6197477!4d-49.3366458?hl=pt-BR>. Acesso em 21 ago. 2019. Research. v. 35, n. 12, p. 1195 -1209, 2017. 
HAASE, R. P. Product/Service-System Origins and Trajectories: A Systematic Literature Review of PSS Definitions and their Characteristics. Procedia CIRP, v. 64, p. 157-162, 1 jan. 2017.

HEISKANEN, E.; JALAS, M. Dematerialization Through Services-A Review and Evaluation of the Debate. p. 436, 2000.

IBPES. THE IPBES REGIONAL ASSESMENT REPORT ON BIODIVERSITY AND ECOSYSTEM SERVICES FOR THE AMERICAS, Intergovernmental Science-Policy Plattform on Biodiversity and Ecosystem Services, p. 491-497, 2018

JAGTAP, S.; RAHIMIFARD, S.; KINGDOM, U. Utilisation of Internet of Things to Improve Resource Efficiency of Food Supply Chains. n. November, p. 8-19, 2017.

KANDA, Y.; NAKAGAMI, Y. What is Product-Service Systems (PSS)-A review on PSS researches and relevant policies. IGES Kansai Research Centre-white paper, n. 1, p. 0-23, 2006.

KUMEGAWA, L. S. CONSÓRCIO INTERMUNICIPAL PARA RESÍDUOS SÓLIDOS URBANOS DE CURITIBA: uma análise do sistema integrado de Processamento e Aproveitamento de Resíduos. 2018. 150f. Dissertação (mestrado) - Programa de Pós-Graduação em Tecnologia e Sociedade, Universidade Tecnológica Federal do Paraná. Curitiba, 2008.

LAMIM-GUEDES, V. Consciência negra, justiça ambiental e sustentabilidade. Sustentabilidade em Debate, v. 3, p. 223-238, 2012.

LWIZA, F. et al. Energy for Sustainable Development Dis-adoption of Household Biogas technologies in Central Uganda. Energy for Sustainable Development, v. 37, p. 124-132, 2017.

MMA. Ministério do Meio Ambiente Política Nacional de Resíduos Sólidos. Disponível em: <http://www.mma.gov.br/política-de-resíduos-sólidos>. Acesso em: 23 set. 2018.

MONT, O. . Clarifying the concept of product-service system. Journal of Cleaner Production, v. 10, n. 3, p. 237-245, jun. 2002.

MONTEIRO, J. Manual de gerenciamento integrado de resíduos sólidos. 2001. 
OLIVEIRA, T.; OLIVEIRA, T. F. Exposição às substâncias cancerígenas no ambiente de trabalho: ameaça a saúde dos catadores de lixo do Brasil. Tempus Actas de Saúde Coletiva, v. 7, n. 2, p. Pág. 139-143, 25 set. 2013.

ORSKOV, E. R. et al. ScienceDirect Overview of holistic application of biogas for small scale farmers in Sub-Saharan Africa. Biomass and Bioenergy, v. 70, p. 4-16, 2014.

ORTIZ, W.; TERRAPON-PFA, J.; DIENST, C. Understanding the diffusion of domestic biogas technologies. Systematic conceptualisation of existing evidence from developing and emerging countries. v. 74, n. September 2016, p. 1287-1299, 2017.

PUBLICADIREITO. As dimensões do desenvolvimento sustentável. Disponível em: <http://www.publicadireito.com.br/artigos/?cod=8b9b3436fc4466e9>. Acesso em: 13 mai. 2019.

RAABE, B. et al. Collaboration Platform for Enabling Industrial Symbiosis: Application of the By-product Exchange Network Model. Procedia CIRP, v. 61, p. 263-268, 1 jan. 2017.

RAMOS, N. F. et al. Desenvolvimento de ferramenta para diagnóstico ambiental de lixões de resíduos sólidos urbanos no Brasil. Engenharia Sanitaria e Ambiental, v. 22, n. 6, p. 1233-1241, dez. 2017.

SACHS, I. Caminhos para o desenvolvimento sustentável. Organização: Paula YoneStroh. Rio de Janeiro: Garamond, 3.ed., 2008.

SANDEC / EAWAG. Solid Waste Management in Developing Countries. Disponível em:

<https://www.eawag.ch/fileadmin/Domain1/Abteilungen/sandec/publikationen/ SWM/General_Overview/Zurbruegg_2002_SWM_DC.pdf>. Acesso em 13 mai. 2019.

SAHIMAA, O. et al. Method for residual household waste composition studies. Waste Management, v. 46, p. 3-14, 1 dez. 2015.

SHAHBAZI, S. et al. Material efficiency in manufacturing: swedish evidence on potential, barriers and strategies. Journal of Cleaner Production, v. 127, p. 438450, 20 jul. 2016. 
SMITH, M. T.; SCHROENN, J.; BLIGNAUT, J. N. The financial and economic feasibility of rural household biodigesters for poor communities in South Africa. Waste Management, v. 34, n. 2, p. 352-362, 2014.

STANCHEV, P. et al. Municipal solid waste management and waste-to-energy in the context of a circular economy and energy recycling in Europe. v. 141, 2017.

TUKKER, A. Eight types of product service system: eight ways to sustainability? Business Strategy and the Environment, v. 13, p. 246-260, 2004.

UNNIKRISHNAN, S.; SINGH, A. Resources, Conservation and Recycling Energy recovery in solid waste management through CDM in India and other countries. "Resources, Conservation \& Recycling", v. 54, n. 10, p. 630-640, 2010.

VAN EWIJK, S.; STEGEMANN, J. A. Limitations of the waste hierarchy for achieving absolute reductions in material throughput. Journal of Cleaner Production, v. 132, p. 122-128, 20 set. 2016.

WCED - World Comission on Environment and Development. Our Common Future. Oxford: Oxford University Press, 1987.

YAZAN, D. M. Constructing joint production chains: An enterprise input-output approach for alternative energy use. Resources, Conservation and Recycling, v. 107, p. 38-52, 1 fev. 2016.

Recebido: 23 out. 2019

Aprovado: $17 \mathrm{dez} .2019$

DOI: $10.3895 /$ rbpd.v9n1.10592

Como citar: CARVALHO, J. P. A.; BORSATO, M. Proposta alternativa para a adequação dos pequenos

municípios à PNRS, baseada em sistema de produto-serviço. R. bras. Planej. Desenv. Curitiba, v. 9, n. 1, p

89-106, jan./abr. 2020. Disponível em: <https://periodicos.utfpr.edu.br/rbpd>. Acesso em: XXX.

Correspondência:

Jairo Pablo Alves de Carvalho

Av. Sete de Setembro, 3165 - Rebouças - Curitiba, PR

Direito autoral: Este artigo está licenciado sob os termos da Licença CreativeCommons-Atribuição 4.0

Internacional. 